\title{
The American Phytopathological Society Elected and Appointed Officers, Representatives, and Committees for 2000-2001
}

Council

President: S. A. Slack

President-Elect: N. T. Keen

Immediate Past President: N. K. Van Alfen

Vice President: J. Fletcher

Secretary: D. J. Jardine

Treasurer: J. L. Sherwood

Editor-in-Chief, Phytopathology: B. I. Hillman

Editor-in-Chief, Plant Disease: A. R. Biggs

Editor-in-Chief, Molecular Plant-Microbe Interactions : H. P. Spaink

Editor-in-Chief, Phytopathology News: R. F. Nyvall

Editor-in-Chief, APS Press: R. C. Ploetz

Senior Councilor-at-Large: R. C. Gergerich

Intermediate Councilor-at-Large: S. S. Hurtt

Junior Councilor-at-Large: J. H. Andrews

Councilor of the Caribbean Division: S. Cantrell

Councilor of the North Central Division: F. W. Nutter Jr.

Councilor of the Northeastern Division: B. J. Christ

Councilor of the Pacific Division: M. E. Matheron

Councilor of the Potomac Division: E. L. Stromberg

Councilor of the Southern Division: D. T. Beadle

Ex offico: APS Foundation Representative: S. M. Coakley

Ex offico: Executive Vice President: S. C. Nelson

\section{Representatives}

American Association for the Advancement of Science: J. Cook

American Institute of Biological Sciences: J. Fletcher

American Type Culture Collection: R. S. Nelson

Coalition for Education in the Life Sciences: M. A. Sulzinski

Coalition for Funding Agricultural Research: S. A. Tolin

Coalition for Research on Plant Systems: S. A. Tolin \& J. L. Sherwood

Council for Agricultural Science and Technology: B. J. Jacobsen

International Society of Arboriculture: R. J. Stipes

International Society for Plant Pathology: Chair: A. M. Alvarez. G. S. Abawi, L.

V. Madden, J. Moyer, C. C. Mundt, B. W. Pennypacker, L. Sequeira, P. S.

Teng, A. H. van Bruggen, R. S. Zeigler

International Union of Microbiological Societies: C. J. Baker

Mycological Society of America: C. M. Stiles

National Committee on Worker Safety Standards: S. A. Alexander

National Institutes for the Environment: T. A. Kucharek

National Research Council, Commission on Life Sciences: D. J. Jardine

National Research Council, CLS/Board of Agriculture: S. A. Slack

Society of Environmental Toxicology and Chemistry: M. Simini

Society of Nematologists: E. L. Davis

APS Historian

P. D. Peterson Jr.

APS Foundation, Board of Directors

Chair: S. M. Coakley. Vice Chair: B. J. Jacobsen. Secretary: D. A. Cooksey

Treasurer: J. R. French. Past Chair: O. W. Barnett. D. E. Mathre, A. O. Paulus,

Ex offcio: J. L. Sherwood, A. E. Glenn, S. C. Nelson

\section{Meetings Board}

Chair: N. T. Keen. Vice Chair: J. Fletcher., R. C. Gergerich, L. D. Dunckle, J. E. Lundquist, F. W. Nutter Jr., P. V. Ouedemans, E. N. Rosskopf, H. B. Scholthof, G. L. Schumann, Staff

\section{National Plant Pathology Board}

Chair: O. W. Barnett. J. Amador, N. B. Carroll, R. J. Cook, J. L. Sherwood, S. A. Tolin, A. K. Vidaver

Office of Electronic Communication (OEC)

Director: J. D. MacDonald. C. T. Bull, A. O. Charkowski, D. M. Eastburn, G. G. Grove, N. Grunwald, G. Hughes, J. B. Ristaino, Ex officio: M. Wimer

Office of International Programs Advisory Board

Director: R. A. Bennett. Immediate Past Director: L. Sequeira. G. S. Abawi, K. F. Cardwell, N. W. Schaad, J. R. Steadman, S. C. Nelson (APS Executive Vice

Publication no. P-2000-1128-010
President), A. O. Paulus (APS Foundation Representative)

Education: Chair: K. F. Cardwell. G. S. Abawi, J. McBeath, R. S. Zeigler

Policies and Program(s): Chair: G. S. Abawi. R. A. Bennett, L. Black, K. F. Cardwell, J. Dubin, N. W. Schaad, J. R. Steadman

Research: Chair: N. W. Schaad. S. H. De Boer, G. A. Forbes, E. R. French,

Service: Chair: J. R. Steadman. W. M. Brown, S. H. De Boer, T. A. Pastor-Corrales, R. Ploetz

OPAE Advisory Board

Director: G. L. Tylka. Immediate Past Director: G. Bergstrom, M. N. Cline, C. Curtis, M. Ellis, B. D. Mogen, J. Ristaino, J. R. Steadman, S. A. Slack, O. W. Barnett, Ex officio: Staff

Communications: Chair: TBD. R. H. Brlansky, L. D. Carsten, M. N. Cline, R. F. Line, N. W. Schaad, P. R. Sellers, Ex offcio: R. F. Nyvall, Staff

APS Publications Board

Chair: B. I. Hillman. A. R. Biggs, H. P. Spaink, R. C. Ploetz, R. F. Nyvall, J. D. MacDonald, T. Murray, G. L. Schumann, Staff (ex officio)

Editors of APS Press

Editor-in-Chief: R. C. Ploetz. Senior Editors: C. M. Becker, D. M. Benson, A Bergamin Filho, E. J. Braun, W. Chun, A. B. Gould, G. Hughes, J. B. Jones, G. W. Moorman, K-B. G. Scholthof, Ex officio: J. L. Sherwood, Staff

APS Press Editorial Boards and Committees

Biological and Cultural Tests: Senior Editor: S. C. Bost. Section Editors: M. J. Boehm, K. L. Bowen, D. R. Cooley, G. J. Holmes, T. S. Isakeit, D. J. Jardine, A. P. Keinath, P. E. Lipps, G. P. Munkvold, J. Williams- Woodward

Fungicide and Nematicide Tests: Senior Editor: R. E. Baird. Section Editors: W. W. Bockus, P. Bristow, L. Datnoff, W. Elmer, A. Hagan, B. Padgett, R. N. Raid, D. Rosenberger, G. Schumann, D. Weingartner, K. Yoder, H. Young

Illustrations of Plant Pathogens and Diseases: Chair: Immediate Past Chair: E. J. Braun. G. J. Holmes, G. W. Hudler, V. Kumar, G. W. Moorman, N. R. O’Neill, J. E. Partridge, A. C. Schilder, G. R. Stanosz, T. J. Volk, J. E. Watkins

Phytopathological Classics: Chair: P. D. Peterson. Immediate Past Chair: T. D. Murray. D. W. Fulbright, C. S. Griffith, C. M. Liddell, K-B. G. Scholthof

Standardization of Common Names of Plant Diseases: Chair: P. F. Bertrand. Vice Chair: TBD. Immediate Past Chair: M. Putnam. C. W. Averre, A. Grybauskas, J. H. Hart, M. E. Matheron, E. V. Podleckis, T. A. Zitter

Editors of MOLECULAR PLANT-MICROBE INTERACTIONS

Editor-in-Chief: H. P. Spaink. Senior Editors: C. A. Boucher, J. Glazebrook, F. Govers, D. Haas, M. J. Harrison, J. W. Kronstad, J. E. Schoelz, J. Stougaard, M. Udvardi, V. M. Williamson. Associate Editors: M. Arlat, D. L. Beck, N. J. Brewin, J. P. Carr, J. Dangl, X. Dong, D. J. Ebbole, G. Gheysen, M. Grant, M. M. Holsters, S. Kamoun, B. N. Kunkel, S. A. Lommel, J. McDowell, K. W. Mendgen, T. Nuernberger, J. E. Parker, S. Perotto, L. S. Pierson III, J. M. Salmeron, S. C. Somerville, J. Sweigard, N. D. Young

\section{Editors of PHYTOPATHOLOGY}

Editor-in-Chief: B. I. Hillman. Senior Editors: D. M. Bird, D. A. Cuppels, D. R. Fravel, R. L. Gilbertson, S. H. Hulbert, A. M. Jarosz, R. S. Nelson, K. V. Subbarao, X.-B. Yang. Associate Editors: D. Andrivon, M. J. Davis, J. A. Duthie, S. E. Gold, T. R. Gordon, N. H. Grimsley, Y. Jin, D. Y. Kobayashi, C. C. Mundt, T. L. Peever, J. M. Raaijmakers, M. J. Roossinck, C. L. Schardl, D. A. Schisler, S. M. Schneider, J. E. Sherwood, D. C. Stenger, L. C. van Loon

\section{Editors of PLANT DISEASE}

Editor-in-Chief: A. R. Biggs. Senior Editors: K. L. Bowen, B. D. Bruton, P. D. Colyer, L. L. Domier, T. C. Harrington, M. K. Hausbeck, P. L. Landschoot, C. H. Liao, G. P. Munkvold, K. L. Stevenson. Feature Editor: N. A. Tisserat. Disease Notes Assigning Editor: A. P. Keinath. Focus Editor: T. Kommedahl. Associate Editors: P. Bonello, B. M. Cunfer, L. J. du Toit, K. C. Eastwell, S. A. Enebak, R. D. Gitaitis, F. A. Gray, R. W. Hammond, T. C. Harrington, J. R. Hartman, D. E. Hershman, R. M. Hunger, L. L. Kinkel, J. S. Lehman, J. P. Noe, J. W. Pscheidt, P. L. Pusey, J. S. Russin, D. A. Samac, D. L. Seifers, L. W. Timmer, J. Y. Uchida, J. P. Wilson, C. P. Woloshuk, G. Y. Yuen

\section{Editors of PLANT HEALTH PROGRESS}

Editor-in-Chief: T. Murray. Senior Editors: A. M. Alvarez, T. J. Gerik, G. G. Grove, K. Moore, H. V. Morton, S. T. Nameth, M .Putnam, K. K. Rane, J. L. Starr, K. L. Steffey, Ex officio: A. P. Keinath, R. C. Ploetz, J. B. Ristaino, M. Wimer 


\section{Editors of THE PLANT HEALTH INSTRUCTOR}

Editor-in-Chief: G. L. Schumann. M. L. Agnew, M. J. Boehm, C. J. D’Arcy, D. M. Eastburn, D. C. Gross, K. B. Johnson, M. L. Powelson, D. L. Schadler, D. O. TeBeest, Ex officio: C. Ash, S. Kronmiller

\section{Society Internal Relations}

Awards and Honors: Chair: I. Barash, Vice Chair: G. Payne, Immediate Past Chair: J. H. Hill, T. J. Burr, L. D. Dunkle, D. C. Gross, W. R. Stevenson Committee on Committees: Chair: R. C. Gergerich, S. S. Hurtt, J. H. Andrews

Financial Advisory: Chair: J. L. Sherwood. J. H. Andrews, J. Fletcher, N. T. Keen, R. C. Ploetz, S. A. Slack, E. L. Stromberg, Ex offcio: Staff

Headquarters Operations: Chair: N. K. Van Alfen, J. L. Sherwood, S. A. Slack, N. T. Keen, AACC representatives, Ex officio: S. C. Nelson

Industry Advisory: Chair: C. M. Becker, N. B. Carroll, M. N. Cline, S. P. Falk, J. R. James, A. J. Julis, R. P. Kaiser, D. F. Kendra, K. T. Kmetz, H. V. Morton, J. L. Riggs, C. L. Sutula

Meeting Site Selection: Chair: S. S. Hurtt, D. T. Beadle, S. Cantrell, B. J. Christ, R. C. Gergerich, M. A. Matheron .F. W. Nutter Jr., E. L. Stromberg, Ex offcio: Staff

Nominating: Chair: S. S. Hurtt, D. T. Beadle, S. Cantrell, B. J. Christ, M. A. Matheron, F. W. Nutter Jr., E. L. Stromberg

Phytopathology News Advisory: Chair: R. Nyvall, J. H. Andrews,

Placement: Chair: E. A. Sobek, Vice Chair: S. L. Walker Immediate Past Chair: D. A. Palmer. C. H. Bock, A. K. Culbreath, L. J. du Toit, R. A. Kuznia. R. Rong, A. K. Wood

Program: Chair: N. T. Keen. Vice Chair: J. Fletcher. Immediate Past Chair: S. A. Slack. R. C. Gergerich, S. C. Nelson, Committee Chairs

Sustaining Associates: Chair: M. N. Cline Immediate Past Chair: P. F. Colbaugh, S. R. Siemer

\section{Society General Policies}

Biotechnology Impact Assessment: Chair: B. K. Duffy, Vice Chair: R. R. Martin. Immediate Past Chair: J. L. Norelli, J. H. Andrews, G. J. Defago, M. R. Finckh, V. Kumar, D. C. Sands, D. C. Thompson, P. Warrior, Y. Yang

Collections and Germ Plasm: Chair: J. C. Rupe. Vice Chair: M. E. Miller Immediate Past Chair: R. F. Line, M. L. Carson, A. G. Gillaspie, J. H. Hill, S. Leath, B. Lovic, E. A. Milus, H. F. Schwartz, C. E. Thomas, A. T. Tschanz, J. P. Wilson

Cultural Diversity: Chair: C. M. Okpodu. Vice Chair: R. L. Grayson, Immediate Past Chair: A. E. Dorrance, M. T. Abdullah, D. G. Fernando, D. M. Huber, T. L. Kirkpatrick, J. E. Lundquist, M. A. Morant, N. Ntahimpera, B. P. Singh, R. H. von Qualen

Extension: Chair: M. A. Draper Vice Chair: D. E. Legard. Immediate Past Chair: M. A. Ellis, E. A. Brown, R. M. Davis, R. L. Forster, D. E. Hershman, T. S. Isakeit, S. R. Koenning, J. S. Lamboy, S. T. Nameth, M. A. Newman, L. P. Pottorff, R. L. Schlub

Graduate Student: Chair: A. E. Glenn Vice Chair: L. M. Sosnoskie Immediate Past Chair: T. K. Mitchell, M. G. Anderson, V. M. Aris, L. E. Hoffman, Z. Kanaan-Atallah, E. M. Martin, L.D. Price, J. D. Reddy, C. Riegel, S. J. Scheuerell, P. M. Sforza

Industry: Chair: C. T. Schiller Vice Chair: K. W. Seebold Immediate Past Chair: E. C. Tedford, N. B. Carroll, S. R. Foor, B. J. Jacobsen, C. S. Morton, C. L. Palmer, J. L. Riggs, R. E. Ross, K. K. Shetty

Private Practice: Chair: C. H. Mellinger. Vice Chair: W. T. Cobb. Vice Chair-Elect: B. Rao. Immediate Past Chair: W. R. Landis, J. H. Hart, H. D. Quemeda, G. A. Snow

Regulatory Plant Pathology: Chair: L. G. Brown Vice Chair: S. D. Cohen. Immediate Past Chair: R. E. Ykema, T. N. Boratynski, K. L. Kosta, A. Mengistu, G. M. Milbrath, C. M. Ocamb, S. A. Rizvi, A. B. Sinderman, A. T. Tschanz, X.-B. Yang

Teaching: Chair: M. B. Riley Vice Chair: M. A. Sulzinski. Immediate Past Chair: T. A. Evans, D. N. Appel, A. B. Baudoin, E. J. Braun, R. B. Carroll, M. J. Clements, C. R. Curtis, K. D. Gwinn, J. C. Locke, K. S. McLean, C. S. Rothrock, A. T. Saad, P. Vincelli

Women in Plant Pathology: Chair: L. E. Hanson Vice Chair: P. D. Adams, Immediate Past Chair: N. Kokalis-Burelle, M. T. Abdullah, M. K. Hausbeck, D. B. Lopes, C. Loschinkohl, L. Ortiz-Ribbing, E. Rosskopf, N. K. Zidack

Youth Program: Chair: R. N. Raid. Vice Chair: K. Shelton. Immediate Past Chair: C. R. Curtis, A. J. Anderson, Y. T. Berthier-Schaad, J. J. Choi, J. Esnard, D. W. Kalb, L. D. Moore, B. L. Randall-Schadel, A. Tally

\section{Subject Matter}

Bacteriology: Chair: F. J. Louws. Vice Chair: C. A. Ishimaru. Immediate Past Chair: L. S. Pierson III, J. D. Barak, G. A. Beattie, S. M. Brumbley, J. C. Chen, R. Frederick, S. Y. He, E. N. Okey, S. Q. Pan, A. R. Poplawsky, K. L. Schroeder, G. W. Sundin

Biochemistry, Physiology, and Molecular Biology: Chair: R. G. Upchurch. Vice Chair: T. J. Wolpert. Immediate Past Chair: C. P. Woloshuk. J. P. Bolar, K. R. Davis, M. B. Dickman, H. Leung, T. K. Mitchell, L. D. Price, S. B. Von
Bodman, M. N. Zook

Biological Control: Chair: C. M. Press. Vice Chair: W. F. Stoneman. Immediate Past Chair: V. O. Stockwell, S. M. Boyetchko, B. K. Duffy, D. GurianSherman, D. M. Huber, N. I. Kahn, L. L. Kinkel, B. B. McSpadden-Gardener, E. N. Rosskopf, D. O. Te Beest, R. S. Utkhede, P. Warrior

Chemical Control: Chair: N. Lalancette. Vice Chair: D. B. Langston. Immediate Past Chair: L. E. Zang, D. T. Beadle, T. B. Brenneman, R. Gold, C. L. Kohls, T. M. Momol, G. Olaya

Diagnostics: Chair: K. K. Rane. Vice Chair: J. W. Beale. Immediate Past Chair: T. C. Stebbins, T.C. Creswell, L. J. Du Toit, S. T. Koike, S. M. Lerner, J. Mullen, M. Putnam, B. Rao, D. L. Scott, K. L. Snover, M. R. Williamson

Diseases of Ornamental Plants: Chair: A. B. Sinderman Vice Chair: G. A. Chastagner, Immediate Past Chair: J. C. Locke, J. W. Buck, D. L. Clement, A. B. Gould, M. K. Hausbeck, G. W. Moorman, S. R. Vann, J. E. Watkins

Environmental Quality and Plant Health: Chair: P. A. Gay. Vice Chair: M. S. Krause. Immediate Past Chair: M. Simini, R. Bulluck, C. R. Curtis, J. Kotcon, M. S. Kraus, J. S. Lamboy, T. Schubert, S. R. Shafer, M. Skaria, G. S. Smith, S. R. Vann

Epidemiology: Chair: W. F. Pfender. Vice Chair: K. A. Garrett. Immediate Past Chair: C. S. Thomas, S. Chakraborty, K. M. Emery, N. Evans, D. G. Fernando, G. A. Forbes, J. J. Hao, S. A. Pethybridge, S. Sanogo, H. Su, B. M. Wu

Forest Pathology: Chair: W. J. Otrosina. Vice Chair: L. M. Haugen. Immediate Past Chair: K. O. Britton, D. Bannwart, L. Bernier, J. N. Bruhn, S. D. Cohen, R. C. Hamelin, R. S. Hunt, J. L. Kerrigan, J. T. Kliejunas, P. C. Spaine, G. R. Stanosz, J. A. Walla, P. J. Zambino

Genetics: Chair: T. L. Peever. Vice Chair: S. E. Gold. Immediate Past Chair: D. M. Francis, S. Kang, G. Kuldau, F. N. Lee, J. S. Lehman, C. M. Liddell, R. J. Nelson, J. B. Rasmussen, G-L. Wang

Host Resistance: Chair: J. A. Thies. Vice Chair: A. E. Dorrance. Immediate Past Chair: J. M. Windes, X. Chen, M. J. Clements, K. L. Crabtree, P. T. Himmel, J. A. Kolmer, C. S. Kousik, G. L. Tylka, I. E. Yates, T. A. Zitter

Integrated Pest Management: Chair: G. L. Schuster. Vice Chair: S. A. Alexander. Immediate Past Chair: G. L. Schuster. W. M. Brown, Jr., A. K. Culbreath, D. S. Egel, L. Fought, B. E. Paulrud, H. Scherm, S. F. Shamoun, G. S. Smith, E. L. Stromberg, N. Zriba

Mycology: Chair: M. A. Cubeta Vice Chair: C. M. Stiles. Immediate Past Chair: F. M. Dugan, R. E. Baird, M. L. Boyd, J. C. Correll, D. M. Eastburn, S. B. Goodwin, H. C. Kistler, D. J. Royse, L. Wasilwa

Mycotoxicology: Chair: G. P. Munkvold. Vice Chair: A. E. Desjardins. Immediate Past Chair: F. Trail, H. K. Abbas, C. W. Bacon, P. J. Cotty, K. E. Damann, J. P. Duvick, N. J. Keller, M. P. McMullen, D. A. Schisler, D. M. Wilson, C. P. Woloshuk

Nematology: Chair: C. S. Johnson. Vice Chair: W. T. Crow. Immediate Past Chair: J. A. LaMondia, D. W. Dickson, J. Esnard, S. A. Lewis, T. L. Niblack, R. D. Riggs, B. P. Singh,

Pathogen Resistance: Chair: P. I. Lewis Vice Chair: T. B. Brenneman. Immediate Past Chair: G. Olaya, K. L. Crabtree, S. N. Jeffers, W. D. Koeller, G. M. Leavitt, P. S. McManus, R. N. Raid, R. A. Romero, F. P. Wong, H. L. Ypema

Phyllosphere Microbiology: Chair: J. Mercier. Vice Chair: C. C. Mundt Immediate Past Chair: H. C. Hoch, D. Aylor, O. Carisse, J. P. Hubbard, B. J. Jacobsen, K. B. Johnson, O. Kilic, S. Lindow, R. L. Nicholson, P. L. Pusey, B. D. Shaw, V. O. Stockwell

Plant Pathogen and Disease Detection: Chair: L. Levy. Vice Chair: C. A. Levesque. Immediate Past Chair: R. R. Martin, V. DeHerrera, N. Glynn, S. N. Jeffers, P. D. Kharbanda, W. H. Sheta, J. Q. Xia

Plant Disease Losses: Chair: S. Chakraborty Vice Chair: W. W. Turechek. Immediate Past Chair: F. W. Nutter Jr., J. C. Comstock, J. Guan, C. A. Hollier, G. Hughes, D. L. Long, M. H. Royer, A. Wrather

Postharvest Pathology: Chair: W. S. Conway. Vice Chair: W. J. Janisiewicz. Immediate Past Chair: J. A. Bartz, J. E. Adaskeveg, B. D. Bruton, G. J. Holmes, E. M. Imai, D. F. Kendra, P. G. Sanderson

Seed Pathology: Chair: C. J. Kurowski. Vice Chair: L. M. Shepherd. Immediate Past Chair: A. M. Alvarez, H. Bouzar, P. Fenn, A. B. Thornton, M. G. Tiffany, A. M. VanBuren, R. R. Walcott, W. L. Wiebe

Soil Microbiology and Root Diseases: Chair: D. R. Fravel. Vice Chair: M. L. Smither-Kopperl. Immediate Past Chair: K. V. Subbarao, R. G. Bhat, C. H. Canady, W. Chen, W. H. Elmer, J. A. Gracia-Garza, P. Lemanceau, R. D. Martyn, S. L. Meyer, J. M. Raaijmakers, K. G. Shetty, N. Shishkoff, A. G. Stone

Tropical Plant Pathology: Chair: R. G. McGuire. Vice Chair: J. V. Da Graca Immediate Past Chair: J. Y. Uchida, S. J. Bagenski, R. Bandyopadhyay, D. R. Greenough, M. P. Grisham, H. A. Harrison, R. F. Lee, A. A. MacNab

Turfgrass Pathology: Chair: M. J. Boehm. Vice Chair: L. J. Stowell. Immediate Past Chair: E. B. Nelson, B. B. Clarke, L. E. Datnoff, K. J. Gard, D. Green, R. X. Latin, E. A. Milus, B. D. Mitchum, J. F. Powell, G. L. Schumann, J. M. Vargas

Virology: Chair: W. M. Wintermantel. Vice Chair:J. F. Murphy. Vice ChairElect: J. E. Schoelz. Immediate Past Chair: K. M. Scheets, V. Bowman-Vance, R. H. Brlansky, J. K. Brown, A. L. Eggenberger, C. J. M. Maroon, K. L. Perry, B. R. Reddick, M. Zaitlin 